\title{
Light gravitinos at colliders and implications for cosmology
}

\author{
Jonathan L. Feng, ${ }^{1}$ Marc Kamionkowski, ${ }^{2}$ and Samuel K. Lee ${ }^{2}$ \\ ${ }^{1}$ Department of Physics and Astronomy, University of California, Irvine, California 92697, USA \\ ${ }^{2}$ California Institute of Technology, Mail Code 350-17, Pasadena, California 91125, USA
}

(Received 23 April 2010; published 28 July 2010)

\begin{abstract}
Light gravitinos, with mass in the $\mathrm{eV}$ to $\mathrm{MeV}$ range, are well motivated in particle physics, but their status as dark-matter candidates is muddled by early-Universe uncertainties. We investigate how upcoming data from colliders may clarify this picture. Light gravitinos are produced primarily in the decays of the next-to-lightest supersymmetric particle, resulting in spectacular signals, including di-photons, delayed and nonpointing photons, kinked charged tracks, and heavy metastable charged particles. We find that the Tevatron with $20 \mathrm{fb}^{-1}$ and the $7 \mathrm{TeV}$ LHC with $1 \mathrm{fb}^{-1}$ may both see evidence for hundreds of light-gravitino events. Remarkably, this collider data is also well suited to distinguish between currently viable light-gravitino scenarios, with striking implications for structure formation, inflation, and other early-Universe cosmology.
\end{abstract}

DOI: 10.1103/PhysRevD.82.015012

\section{INTRODUCTION}

Supersymmetry is one of the most promising ideas for new physics beyond the standard model. Supersymmetric theories that incorporate local supersymmetry (or supergravity) predict the existence of the gravitino, the spin- $3 / 2$ superpartner of the graviton. When supersymmetry is broken, the gravitino acquires a mass through the super-Higgs mechanism, "eating" the spin- $1 / 2$ goldstino, the Goldstone fermion associated with spontaneously broken local supersymmetry [1-4]. In contrast to other superpartners, the gravitino can have a mass $m_{\tilde{G}}$ that is not at the weak scale $m_{\text {weak }} \sim 100 \mathrm{GeV}-1 \mathrm{TeV}$, and viable models exist for gravitino masses as low as the $\mathrm{eV}$ scale and as high as $100 \mathrm{TeV}$. In this work, we consider light gravitinos, with mass in the $\mathrm{eV}$ to $\mathrm{MeV}$ range. Such gravitinos are highly motivated in particle physics, as they emerge in models with gauge-mediated supersymmetry breaking (GMSB), in which constraints on flavor violation are naturally satisfied [5-10].

Light gravitinos also have cosmological motivations. In particular, they are the original supersymmetric darkmatter candidate [11]. Assuming a high reheating temperature, gravitinos are initially in thermal equilibrium and then freeze out while still relativistic. As we discuss in detail below, their resulting relic density is

$$
\Omega_{\tilde{G}} h^{2} \simeq\left[\frac{m_{\tilde{G}}}{1 \mathrm{keV}}\right]\left[\frac{106.75}{g_{* S, f}}\right],
$$

where $g_{* S, f}$ is the number of relativistic degrees of freedom at freeze out, and has been normalized to the total number of degrees of freedom in the standard model. When originally proposed in the 1980s, uncertainties in $h$ and the total matter relic density allowed $m_{\tilde{G}} \sim \mathrm{keV}$. This led to a simple and attractive gravitino-dark-matter scenario, consistent with standard big bang cosmology, in which the
PACS numbers: 14.80.Ly, 13.85.-t, 95.35.+d, 98.80.Cq

Universe cooled from some high temperature, and $\mathrm{keV}$ gravitinos froze out and now form all of the dark matter.

In the intervening years, however, a variety of astrophysical constraints have greatly complicated this picture. First, the dark-matter relic density is now known to be $\Omega_{\mathrm{DM}} h^{2} \simeq 0.11$. Second, constraints on structure formation, as probed by galaxy surveys and Lyman- $\alpha$ forest observations, require that the bulk of dark matter be cold or warm [12]. As we will discuss more fully below, this leads to three scenarios of interest:

(1) $m_{\tilde{G}} \lesssim 15-30 \mathrm{eV}$ : Gravitinos are produced by the standard cosmology leading to Eq. (1); they are hot dark matter, but their contribution is small enough to be consistent with the observed smallscale structure. Some other dark-matter particle is required.

(2) $15-30 \mathrm{eV} \lesssim m_{\tilde{G}} \lesssim$ few $\mathrm{keV}$ : Nonstandard cosmology and a nonstandard gravitino production mechanism are required, both to avoid overclosure and to cool the gravitinos to satisfy small-scale-structure constraints. Some other dark-matter particle may be required.

(3) $m_{\tilde{G}} \gtrsim$ few keV: Nonstandard cosmology is required to dilute the thermal relic density of Eq. (1). Gravitinos produced by thermal freeze out are cold enough to be all of the dark matter.

Note that the original "keV gravitino" scenario, previously favored, is now the most disfavored, in the sense that it is excluded by both overclosure and small-scale-structure constraints. All of the possibilities are rather complicated, however, as in each case, some additional physics is required, either to provide the rest of the dark matter or to modify the history of the early Universe to allow gravitinos to be all of the dark matter.

In this paper, we discuss how collider data may help clarify this picture. Light gravitinos are primarily produced 
at colliders in the decays of the next-to-lightest supersymmetric particle (NLSP). It is a remarkable coincidence that modern particle detectors, with components placed between $1 \mathrm{~cm}$ to $10 \mathrm{~m}$ from the beamline, are beautifully suited to distinguish between the NLSP decay lengths predicted in scenarios 1, 2, and 3. For example, the decay length of a bino NLSP decaying to a gravitino is [13]

$$
c \tau \simeq 23 \mathrm{~cm}\left[\frac{m_{\tilde{G}}}{100 \mathrm{eV}}\right]^{2}\left[\frac{100 \mathrm{GeV}}{m_{\tilde{B}}}\right]^{5} .
$$

This implies that scenarios 1,2 , and 3 make distinct predictions for collider phenomenology, and the identification of the gravitino collider signatures realized in nature may have far-reaching implications for the early Universe.

Of course, this requires that gravitinos can be produced in sufficient numbers and distinguished from standard model backgrounds. In this work, we determine event rates for a variety of signatures, including prompt di-photons and delayed and nonpointing photons (relevant for neutralino-NLSP scenarios), as well as kinked charged tracks and heavy metastable charged particles (relevant for stau-NLSP scenarios). We present results for an assumed final Tevatron dataset $\left(20 \mathrm{fb}^{-1}\right.$ of $2 \mathrm{TeV} p \bar{p}$ collisions), an early LHC dataset $\left(1 \mathrm{fb}^{-1}\right.$ of $7 \mathrm{TeV} p p$ collisions), and a future LHC dataset $\left(10 \mathrm{fb}^{-1}\right.$ of $14 \mathrm{TeV}$ $p p$ collisions). We find that the final Tevatron and early LHC data have roughly equivalent sensitivity to these events, with both capable of seeing hundreds of distinctive light-gravitino events. The full LHC data greatly extends the reach in parameter space, and may also allow precision measurements of NLSP lifetimes and gravitino masses.

We begin in Sec. II by reviewing the cosmological bounds on light gravitinos and discussing how these bounds are relaxed in early-Universe scenarios that differ from the canonical one. In Sec. III we then discuss NLSP decays to gravitinos, GMSB models, and current collider constraints. In Sec. IV we present our results for the number of light-gravitino events at colliders, based on collider simulations, and discuss the cosmological implications. We summarize our conclusions in Sec. V.

\section{LIGHT-GRAVITINO COSMOLOGY}

\section{A. Canonical scenario}

\section{Relic abundance}

In the currently canonical scenario, after inflation, the Universe is reheated to a temperature $T_{R}$ that is assumed to be far higher (e.g., $10^{12}$ or $10^{15} \mathrm{GeV}$ ) than the weak scale. During this phase, inelastic scattering processes and decays can convert standard model particles in the thermal bath into gravitinos [14-18]. The rate $C_{\tilde{G}}$ per unit volume for production of light gravitinos (strictly speaking, only the spin- $1 / 2$ goldstino components) can be calculated by considering all such processes, which primarily involve strong [19] and electroweak gauge bosons [20,21], as well as top quarks [22]. The total result, valid in the limit $T \gg m_{\mathrm{SUSY}}$, where $m_{\mathrm{SUSY}}$ is the scale of the superpartner masses, is [22]

$$
C_{\tilde{G}} \simeq 15 \frac{m_{\tilde{g}}^{2}}{m_{\tilde{G}}^{2}} \frac{T^{6}}{M_{\mathrm{pl}}^{2}},
$$

where $M_{\mathrm{pl}} \simeq 1.2 \times 10^{19} \mathrm{GeV}$ is the Planck mass. Here we have assumed that the gaugino masses $m_{\tilde{g}, 1}, m_{\tilde{g}, 2}$, and $m_{\tilde{g}, 3}$ and the trilinear scalar coupling $A_{t}$ are at a common mass scale. For simplicity, we have set them equal to a universal gaugino mass $m_{\tilde{g}}$.

The evolution of the gravitino number density $n_{\tilde{G}}$ via these production processes, and their inverses, is governed by the Boltzmann equation

$$
\frac{d n_{\tilde{G}}}{d t}+3 H n_{\tilde{G}}=C_{\tilde{G}}-\Gamma n_{\tilde{G}},
$$

where $H$ is the Hubble expansion rate and $\Gamma$ is the rate of processes that annihilate gravitinos. The $3 H n_{\tilde{G}}$ term accounts for dilution of the number density due to cosmological expansion. If $\Gamma \gg H$, gravitinos are in thermal equilibrium, $\Gamma n_{\tilde{G}}=C_{\tilde{G}}$, and their number density (the solution to the Boltzmann equation) is

$$
n_{\tilde{G}}^{\mathrm{eq}}=g \frac{2 \zeta(3)}{\pi^{2}} T^{3} \simeq 0.24 T^{3} .
$$

Here we used $g=2$, since it is primarily the spin- $1 / 2$ goldstino components that are produced thermally.

The rate $\Gamma$ at which a given gravitino is destroyed in the plasma is then

$$
\Gamma=\frac{C_{\tilde{G}}}{n_{\tilde{G}}^{\mathrm{eq}}} \simeq 60 \frac{m_{\tilde{g}}^{2} T^{3}}{m_{\tilde{G}}^{2} M_{\mathrm{pl}}^{2}} .
$$

Since $\Gamma \propto T^{3}$ and $H \propto T^{2}$, the ratio $\Gamma / H \propto T$ is largest at the highest temperatures. Thus, if $\Gamma\left(T_{R}\right) \geqslant H\left(T_{R}\right)$ at reheating, then gravitinos come into thermal equilibrium shortly after reheating. During this era, the expansion rate is given by $H \simeq 1.66 g_{*}^{1 / 2} T^{2} / M_{\mathrm{pl}}$; assuming reheating temperatures $T_{R} \gg \mathrm{TeV}$, at which all particles in the minimal supersymmetric standard model (MSSM) are relativistic, we set the number $g_{*}$ of relativistic degrees of freedom to $g_{*}\left(T_{R}\right) \simeq 228.75$. Comparing $\Gamma\left(T_{R}\right)$ and $H\left(T_{R}\right)$, we then see that if the reheating temperature satisfies

$$
T_{R} \gtrsim T_{f} \equiv 5 \mathrm{GeV}\left[\frac{m_{\tilde{G}}}{\mathrm{keV}}\right]^{2}\left[\frac{\mathrm{TeV}}{m_{\tilde{g}}}\right]^{2},
$$

then gravitinos come into thermal equilibrium after reheating. Recalling that the production rate, Eq. (3), used here is valid only for $T \gg m_{\mathrm{NLSP}}$ (i.e., $T \gtrsim 10 \mathrm{TeV}$ ), we conclude that for weak-scale gluino masses, light gravitinos with $m_{\tilde{G}} \lesssim \mathrm{MeV}$ will come into thermal equilibrium if the reheating temperature is $T_{R} \gtrsim 5 \times 10^{6} \mathrm{GeV}$.

The creation/annihilation rates for gravitinos at temperatures $T \lesssim 10 \mathrm{TeV}$ have not yet been calculated, and so the 
precise temperature at which gravitinos freeze out (which occurs when $\Gamma \simeq H$ ) cannot yet be determined. Still, gravitinos are produced and destroyed individually, requiring (from $R$-parity conservation) that each creation/destruction is accompanied by creation/destruction of some other supersymmetric particle. Therefore, the freeze-out temperature $T_{f}$ cannot be much lower than the mass $m_{\mathrm{NLSP}}$ of the NLSP, as the equilibrium abundance of SUSY particles then decreases exponentially. We thus conclude that the freeze-out temperature for light gravitinos falls roughly in the range $10 \mathrm{GeV} \lesssim T_{f} \lesssim 10 \mathrm{TeV}$.

With this range of freeze-out temperatures, $T_{f} \gg m_{\tilde{G}}$, so gravitinos are relativistic when they freeze out. The relic gravitino density is then [11]

$$
\Omega_{\tilde{G}} h^{2} \simeq 0.1\left[\frac{m_{\tilde{G}}}{100 \mathrm{eV}}\right]\left[\frac{106.75}{g_{* S, f}}\right],
$$

the standard result for hot relics with $g=2$, where $g_{* S, f}$ is the number of relativistic degrees of freedom when the gravitinos freeze out. If gravitinos freeze out when all of the MSSM degrees of freedom are relativistic, $T_{f} \gg$ $m_{\mathrm{SUSY}}$, and $g_{* S, f}=228.75$. However, it is more likely that freeze out occurs at $T \sim m_{\mathrm{NLSP}} \sim 100 \mathrm{GeV}$, when $g_{* S, f} \sim 100[23]$.

\section{Cosmological constraints}

Given that current cosmic microwave background and structure-formation measurements constrain the density of dark matter to be $\Omega_{\mathrm{DM}} h^{2} \simeq 0.11$, Eq. (8) implies an upper bound $m_{\tilde{G}} \lesssim 200 \mathrm{eV}$. The upper limit $m_{\tilde{G}} \simeq 200 \mathrm{eV}$ is saturated if the gravitino makes up all of the dark matter and freeze-out occurs when $g_{* S, f}=228.75$ is the maximum value allowed in the MSSM. However, a gravitino of this mass would be hot dark matter. It would smooth density perturbations on scales probed by galaxy surveys and the Lyman- $\alpha$ forest to a degree that is highly inconsistent with the data. As $m_{\tilde{G}}$ is reduced from this upper limit, the smoothing scale is increased (the gravitinos get "hotter"), but the gravitino abundance is reduced, thus making the magnitude of the smoothing smaller. A combination of data from the cosmic microwave background, galaxy surveys, and the Lyman- $\alpha$ forest constrain the contribution of a hot component of dark matter to be $\lesssim 15 \%$ [12], implying for $g_{* S, f} \simeq 100$ that $m_{\tilde{G}} \lesssim 15 \mathrm{eV}$. This suggests that the most conservative upper bound is given by $m_{\tilde{G}} \lesssim 30 \mathrm{eV}$, in the case that $g_{* S, f} \simeq 200$ approaches the maximal value allowed in the MSSM. Therefore, in this canonical scenario, thermal gravitinos with mass less than $30 \mathrm{eV}$ make up only a fraction of the dark matter, thus requiring some other particle to be the cold dark matter. This is the first cosmological scenario listed in Sec. I.

We conclude by noting that future astrophysical data are likely to improve. And while the current sensitivity is to gravitino masses as small as $15-30 \mathrm{eV}$, it is forecast that next-generation experiments may be sensitive to gravitino masses as small as $1 \mathrm{eV}$ [24]. A collider detection of a gravitino in the mass range $m_{\tilde{G}}=1-30 \mathrm{eV}$ would thus lead to testable consequences in forthcoming cosmological data.

\section{B. Nonstandard early-Universe scenarios}

There are several ways in which the early-Universe production of relic gravitinos could differ from the canonical scenario outlined above. Thus, there are scenarios in which a gravitino of mass $m_{\tilde{G}} \gtrsim 30 \mathrm{eV}$, ruled out in the canonical model, could be cosmologically consistent or, better yet, completely compose the dark matter.

Let us first consider scenarios in which the gravitinos reach thermal equilibrium in the early Universe, since most observational constraints are strictly valid only under this assumption. As mentioned above, if we only consider particles in the MSSM, then $g_{* S, f} \leq 228.75$, and Eq. (8) suggests an upper limit of $m_{\tilde{G}} \lesssim 200 \mathrm{eV}$ from the relic abundance constraint. One way to evade this limit is to simply consider higher values of $g_{* S, f}$; i.e., gravitinos decouple and freeze out earlier than in the canonical scenario. This may be possible in models with more degrees of freedom than the MSSM. More massive gravitinos that decouple earlier may then be viable, if they have an abundance that obeys the constraint $\Omega_{\mathrm{DM}} h^{2} \lesssim 0.11$.

It is possible that this constraint is saturated and that these heavier gravitinos entirely compose the dark matter. Of course, we must still require that these heavier gravitinos are not so hot as to erase structure to a degree that contradicts observations. The same combination of cosmic microwave background, galaxy-survey, and Lyman- $\alpha$ forest data that was used to constrain $m_{\tilde{G}} \lesssim 30 \mathrm{eV}$ in the canonical scenario can also be used to constrain the gravitino mass in this early-decoupling scenario, assuming that thermal gravitinos make up all of the dark matter. With this assumption, Ref. [12] finds $m_{\tilde{G}} \gtrsim 550 \mathrm{eV}$, using a selection of Lyman- $\alpha$ data. The same authors later find a stronger constraint, $m_{\tilde{G}} \gtrsim 2 \mathrm{keV}$, with Sloan Digital Sky Survey Lyman- $\alpha$ data $[25,26]$, a result slightly weaker than a bound on warm dark-matter models obtained by Ref. [27]. A number of other small-scale observations also seem to support that $m_{\tilde{G}} \gtrsim$ few $\mathrm{keV}$ under these assumptions [28].

We thus conclude that if $30 \mathrm{eV} \lesssim m_{\tilde{G}} \lesssim$ few $\mathrm{keV}$, then thermal gravitinos are too warm to be the only component of the dark matter, regardless of whether or not they have the correct abundance. Gravitinos in this mass range would only be viable if some other nonstandard early-Universe process cools them, or if there is an additional cold component. This is the second scenario mentioned in Sec. I. However, if $m_{\tilde{G}} \gtrsim$ few $\mathrm{keV}$, then gravitinos may be sufficiently cold, and may, in early decoupling scenarios, have the right abundance, to be the dark matter. This is the third scenario outlined in Sec. I. 
Of course, aside from early decoupling, there are other nonstandard mechanisms that can reduce the gravitino abundance. For example, recall that we have no empirical constraints to the early Universe prior to the epoch of bigbang nucleosynthesis (BBN), at which $T \geqslant$ few $\mathrm{MeV}$ [29]. Thus, some entropy-producing process prior to BBN could also dilute the gravitino abundance. It is possible that there may be some exotic early-Universe physics that conspires to produce the same effect. One relatively simple possibility is that the reheating temperature is low. If the reheating temperature is smaller than the freeze-out temperature, then gravitinos will never come into thermal equilibrium, and their relic abundance will thus be accordingly smaller [30-36]. The only catch is that for the light gravitinos we consider here, the reheating temperature must be unusually low for this to occur. For example, if $m_{\tilde{G}}=\mathrm{keV}$ and $m_{\tilde{g}}=$ $300 \mathrm{GeV}$, then Eq. (7) suggests that the reheating temperature must be $T_{R} \lesssim 50 \mathrm{GeV}$. However, recall that this estimate may not be strictly valid at $T \lesssim 10 \mathrm{TeV}$, as we have already noted. Thus, a more careful calculation of the production rate of light gravitinos at low reheating temperatures may be necessary. Nevertheless, such low reheating temperatures have been considered [37], and Ref. [38] has examined an explicit low-reheat scenario in which a gravitino of mass $m_{\tilde{G}}=1-15 \mathrm{keV}$ can have the right abundance to be the dark matter.

Finally, we also note that there may be additional mechanisms affecting the generation of gravitinos. For example, in our discussion we have neglected the nonthermal contribution to the gravitino abundance from out-of-equilibrium decays of other supersymmetric particles. There may also be other significant modes of gravitino production or dilution, including processes involving the messenger particles responsible for GMSB [39-44], nonthermal production via oscillations of the inflaton field [45], $Q$-ball decays [46], and various other mechanisms [47]. There may thus be other reasons why the gravitino abundance or temperature differs from those in the canonical thermal-production scenario; this may be true even if $m_{\tilde{G}} \lesssim 30 \mathrm{eV}$.

To summarize, in the canonical model, gravitinos are required to have mass $m_{\tilde{G}} \lesssim 30 \mathrm{eV}$ and form only a fraction of the dark matter. Gravitinos with mass range $m_{\tilde{G}} \gtrsim$ $30 \mathrm{eV}$ would require nonstandard physics or cosmology to reduce their abundance or temperature to agree with observations. Below we discuss collider signatures of light gravitinos. We close here by noting that such collider data may, if gravitinos are discovered, thus help discriminate between the diversity of early-Universe scenarios for gravitino production.

\section{LIGHT GRAVITINOS AT COLLIDERS}

\section{A. Mass and interactions}

The gravitino mass is determined by the super-Higgs mechanism. In simple models, it is given in terms of the supersymmetry-breaking scale $F$, which has mass dimension 2 , as

$$
m_{\tilde{G}}=\frac{F}{\sqrt{3} M_{*}} \simeq 240 \mathrm{eV}\left[\frac{\sqrt{F}}{10^{3} \mathrm{TeV}}\right]^{2},
$$

where $M_{*} \equiv M_{\mathrm{pl}} / \sqrt{8 \pi} \simeq 2.4 \times 10^{18} \mathrm{GeV}$ is the reduced Planck mass.

The interactions of weak-scale gravitinos are of gravitational strength, as expected since they are the superpartners of gravitons. However, the couplings of the goldstino are proportional to $1 / F[48,49]$. The interactions of light gravitinos are therefore dominated by their goldstino components, and may be much stronger than gravitational. Decays to gravitinos are faster for light gravitinos.

For reasons to be discussed below, we will focus on cases where the NLSP is either the neutralino or the stau. For a neutralino NLSP that is dominantly a bino, the decay widths to gravitinos are [13,50]

$$
\begin{gathered}
\Gamma(\tilde{B} \rightarrow \gamma \tilde{G})=\frac{\cos ^{2} \theta_{W} m_{\tilde{B}}^{5}}{16 \pi F^{2}}, \\
\Gamma(\tilde{B} \rightarrow Z \tilde{G})=\frac{\sin ^{2} \theta_{W} m_{\tilde{B}}^{5}}{16 \pi F^{2}}\left[1-\frac{m_{Z}^{2}}{m_{\tilde{B}}^{2}}\right]^{4},
\end{gathered}
$$

where $\theta_{W}$ is the weak mixing angle. For $m_{\tilde{B}} \lesssim m_{Z}$, decays to $Z$ bosons are negligible or kinematically forbidden, and the corresponding decay length is

$$
c \tau \simeq 23 \mathrm{~cm}\left[\frac{m_{\tilde{G}}}{100 \mathrm{eV}}\right]^{2}\left[\frac{100 \mathrm{GeV}}{m_{\tilde{B}}}\right]^{5} .
$$

For heavier neutralinos, the $Z$ mode may be significant; for very heavy binos, the branching ratio for this mode is $B(Z) \simeq \sin ^{2} \theta_{W} \simeq 0.23$. Decays to $l^{+} l^{-} \tilde{G}$, where $l=$ $(e, \mu$, or $\tau)$ is a charged lepton, and $h \tilde{G}$ may also be possible; however, these modes have branching ratios of $\sim 0.01$ and $\sim 10^{-6}$, respectively.

For stau NLSPs, the decay width is [51]

$$
\Gamma(\tilde{\tau} \rightarrow \tau \tilde{G})=\frac{m_{\tilde{\tau}}^{5}}{16 \pi F^{2}},
$$

corresponding to a decay length

$$
c \tau \simeq 18 \mathrm{~cm}\left[\frac{m_{\tilde{G}}}{100 \mathrm{eV}}\right]^{2}\left[\frac{100 \mathrm{GeV}}{m_{\tilde{\tau}}}\right]^{5} .
$$

As anticipated, in both the neutralino-NLSP and stauNLSP scenarios, the decay lengths for gravitinos in the cosmologically interesting range correspond to distances that bracket the size of collider detectors.

\section{B. GMSB models}

Light gravitinos are expected to be dominantly produced at colliders in the cascade decays of strongly-interacting superpartners, such as squarks and gluinos. Collider con- 
straints therefore depend on the full superpartner spectrum, and so are model dependent. Following most of the literature, we will work in the framework of minimal GMSB, and so we briefly review its features here.

Typical GMSB models are characterized by a hidden sector, a messenger sector, and a visible sector, the MSSM. Supersymmetry breaking is triggered by a hidden-sector gauge-singlet superfield $S$ acquiring the vacuum expectation value $S=M+\theta^{2} F_{S}$. This then generates masses for the messenger-sector fields $M_{\text {mess }}=\lambda M$, where $\lambda$ is a coupling in the superpotential. These in turn generate masses for the visible-sector superpartners that are roughly a loop factor times $\Lambda \equiv F_{S} / M$, and so $\Lambda \sim 100 \mathrm{TeV}$. Note that $M_{\text {mess }}>\Lambda$ is generally assumed.

In the minimal GMSB framework, the entire superpartner spectrum is specified by the parameters

$$
\Lambda, \quad M_{\text {mess }}, \quad N_{5}, \quad \tan \beta, \quad \operatorname{sgn}(\mu), \quad c_{\text {grav }} \text {. }
$$

Here, $\Lambda$ and $M_{\text {mess }}$ are as described above; masses and couplings are generated at $M_{\text {mess }}$ and then evolved to the weak scale via the renormalization group. The number of messenger superfields is given by $N_{5}$, the effective number of $\mathbf{5}+\overline{\mathbf{5}}$ representations of $\mathrm{SU}(5)$. The Higgs sector is specified by the usual parameters $\tan \beta$ and $\operatorname{sgn}(\mu)$. The last parameter is

$$
c_{\text {grav }} \equiv \frac{F}{\lambda F_{S}},
$$

where $F=\left(F_{S}^{2}+\sum_{i} F_{i}^{2}\right)^{1 / 2}$ is the total supersymmetrybreaking vacuum expectation value, which appears in Eq. (9). These relations imply

$$
m_{\tilde{G}}=c_{\text {grav }} \frac{M_{\text {mess }} \Lambda}{\sqrt{3} M_{*}} .
$$

We expect $c_{\text {grav }} \geq 1$, since $F \geq F_{S}$ and $\lambda \lesssim 1$, and in the minimal case that there is only one nonzero $F$-term, we expect $c_{\text {grav }} \sim 1$.

The superpartner masses are determined by the parameters of Eq. (15); for details, see Ref. [52]. Here we note only two things. First, the superpartner masses are determined by gauge couplings. Thus, although, for example, chargino [53] and sneutrino [54] NLSPs have been considered, the canonical NLSP candidates are those with only hypercharge interactions, namely, the bino and righthanded sleptons. Among the right-handed sleptons, the stau is typically the lightest, as renormalization-group evolution and left-right-mixing effects both decrease the stau mass relative to the selectron and smuon, and so we will focus on the bino-NLSP and stau-NLSP scenarios. ${ }^{1}$ Second, the bino and stau masses are proportional to $N_{5}$

\footnotetext{
${ }^{1}$ Note that in the "slepton co-NLSP" scenario, where the three charged sleptons are degenerate to within the mass of the tau, the number of $\tilde{e} \rightarrow e \tilde{G}$ and $\tilde{\mu} \rightarrow \mu \tilde{G}$ decays may be comparable to that of the $\tilde{\tau} \rightarrow \tau \tilde{G}$ decay that usually dominates gravitino production.
}

and $\sqrt{N_{5}}$, respectively. For $N_{5}=1$, the NLSP is the bino in minimal GMSB, but for $N_{5}>1$, the stau may also be the NLSP; see, for example, Fig. 1 of Ref. [51].

Thus, to study the bino-like neutralino-NLSP scenario, we will choose $N_{5}=1$; likewise, we choose $N_{5}=4$ to study the stau-NLSP scenario. For both scenarios, we fix $\tan \beta=20, \mu>0$, and $c_{\text {grav }}=1$. We let $\Lambda$ and $M_{\text {mess }}$ be free parameters. Note that the overall mass scale of the supersymmetric partners is roughly proportional to $\Lambda$, while the gravitino mass depends on both $\Lambda$ and $M_{\text {mess }}$ as in Eq. (17). Thus, scanning over the free GMSB parameters will allow us to explore collider signals for a range of masses. We shall now examine the existing collider constraints on the parameter spaces of these two scenarios.

\section{Current collider constraints}

The high-energy collider signals of GMSB and gravitinos are well studied [50,51,55-69]; for a review of current bounds, see Ref. [70]. Here we summarize the most relevant results for the models and signals we consider below.

We shall discuss GMSB signals in more detail below, but we summarize them briefly here. In the neutralino-NLSP scenario, there are several possible signals. For short-lived neutralinos, nearly all supersymmetry events include two prompt high-energy photons. For longer-lived neutralinos that travel a macroscopic distance before decaying to photons in the detector, delayed or nonprompt photons are possible. The stau-NLSP scenario may also lead to a variety of signatures, depending on the stau lifetime, including acoplanar leptons, tracks with large impact parameters, kinked charged tracks, and heavy metastable charged particles.

Several studies have attempted to place constraints on GMSB models by searching for these signals. Given that we will scan over a large range of the GMSB parameter space, we are primarily interested in constraints that are generally valid over this entire range. We shall thus focus on limits from LEP studies, based on an integrated luminosity of $628 \mathrm{pb}^{-1}$ at center-of-mass energies of 189$209 \mathrm{GeV}$, which combined searches for both GMSB and neutral-Higgs signals [71,72]. The relevant results for our models are the lower limits of $\Lambda \geq 70 \mathrm{TeV}$ for our neutralino-NLSP model, and $\Lambda \gtrsim 20 \mathrm{TeV}$ for our stauNLSP model; see Fig. 6 of Ref. [71]. These constraints on $\Lambda$ are valid for all values of $M_{\text {mess }}$ we include in our scan. Therefore, the allowed region of $M_{\text {mess }}-\Lambda$ parameter space is constrained by these LEP bounds.

However, there are also a number of studies that focused on constraining specific benchmark models [73], which occupy certain points or lines in the GMSB parameter space. Although these constraints cannot be directly applied to our models, we discuss them to get an idea of the robustness of the LEP bounds on our parameter space.

Of these benchmark-model constraints, the best collider bounds on di-photon events are from the Tevatron, includ- 
ing a D0 search based on an integrated luminosity of $1.1 \mathrm{fb}^{-1}$ [74] and a CDF search based on $2.6 \mathrm{fb}^{-1}$ [75]. The D0 and CDF bounds, when interpreted assuming the benchmark GMSB model SPS 8 from Ref. [73], lead to lower bounds on the bino mass of $125 \mathrm{GeV}$ and $150 \mathrm{GeV}$, respectively. For longer-lived neutralinos that travel a macroscopic distance before decaying to photons in the detector, a CDF search for delayed photons, based on $570 \mathrm{pb}^{-1}$ of data, established lower bounds on $m_{\tilde{B}}$ from 70 to $100 \mathrm{GeV}$ for neutralino decay lengths between $20 \mathrm{~cm}$ and $6 \mathrm{~m}$, again when interpreted in the context of SPS 8 [76].

Searches for heavy metastable charged particles have also been performed at D0 [77], assuming the benchmark GMSB model SPS 7 from Ref. [73]. A similar search was performed at CDF [78], but did not interpret results in the context of GMSB models. Based on $\sim 1 \mathrm{fb}^{-1}$ of data, and assuming only Drell-Yan slepton production, the constraints resulting from these two searches are not competitive with the LEP bounds stated previously.

Thus, we shall take the more general LEP bounds as constraints on the two models we consider in this work, and shall further take only conservative values of the lower limits. For the neutralino-NLSP model, we shall only scan the parameter space with $\Lambda \geq 80 \mathrm{TeV}$, which should be comfortably allowed by the LEP bounds. However, we acknowledge that it is possible that the Tevatron data may exclude a small range of NLSP masses within this parameter space comparable to that ruled out in the benchmark model (i.e., $\lesssim 150 \mathrm{GeV}$ ), should this data be reanalyzed in the context of our models. For the stau-NLSP model, we shall scan over $\Lambda \geq 30 \mathrm{TeV}$. Given that the current Tevatron constraints are not competitive with the LEP bounds, all of this parameter space should be allowed. As we will see, hadron colliders have bright prospects for probing the parameter spaces of these models.

\section{TEVATRON AND LHC PROSPECTS}

\section{A. Gravitino signals}

The collider signal of a supersymmetric particle decaying to a gravitino can be classified by (1) the distance from the interaction point at which the decay occurs, and (2) the nature of the accompanying standard model decay products. The former is determined by the gravitino mass and the masses of the decaying supersymmetric particles, as well as the speed with which the decaying particles are produced. The latter is determined primarily by the nature of the NLSP. We shall define and investigate the following categories of events:

(1) Prompt di-photons (in neutralino-NLSP models): Events in which two photons are produced (via a pair of neutralino decays to gravitinos) within $d_{\mathrm{pr}}$ of the interaction point. We take $d_{\mathrm{pr}}=1 \mathrm{~cm}$ as a conservative estimate of the distance to which the origin of any photon can be resolved in detectors at the Tevatron and the LHC. Note that here and below, we cut on the total distance traveled by the NLSP before it decays, not its (transverse) distance from the beamline when it decays.

(2) Nonprompt photons (neutralino NLSP): Events in which at least one photon is produced at a middetector distance $d_{\text {decay }}$ away from the interaction point, where $d_{\mathrm{pr}} \leq d_{\text {decay }} \leq d_{\mathrm{np}}$, and $d_{\mathrm{np}}$ is the maximum distance from the interaction point at which a photon can be observed. We conservatively take $d_{\mathrm{np}}=3 \mathrm{~m}$, roughly the outer radius of the hadronic calorimeters at both the Tevatron and the LHC. (Note that although the calorimeters in the ATLAS detector at the LHC actually extend to $\sim 4 \mathrm{~m}$, those in the CMS detector only extend to $\sim 3 \mathrm{~m}$; we have thus taken the more conservative $3 \mathrm{~m}$ as our cut.) Photons may also convert and be seen in the muon chambers, extending the sensitivity to decays $\sim 10 \mathrm{~m}$ from the interaction point, but we neglect this possibility here. Here we also take $d_{\mathrm{pr}}=1 \mathrm{~cm}$.

Note that this category of events encompasses both nonpointing photons and delayed photons. A nonpointing photon is simply a photon that does not spatially point back to the interaction point. A delayed photon has the further distinction of being produced only after a significant temporal delay following the time of the initial collision. This may occur when the particle that decays to the photon is produced with a low speed, so that it takes a non-negligible amount of time to travel away from the interaction point before it decays. If this amount of time is comparable to the time between collision events, it may be difficult to properly identify the delayed photon with its originating event.

(3) Nonprompt leptons (stau NLSP): Events in which at least one charged lepton is produced (via chargedslepton decays to gravitinos) at a mid-detector distance $d_{\text {decay }}$, where $d_{\text {pr }} \leq d_{\text {decay }} \leq d_{\text {np }}$ as before. We take $d_{\mathrm{np}}=5 \mathrm{~m}$ and $d_{\mathrm{np}}=7 \mathrm{~m}$ as the outer radii of the muon chambers in the detectors at the Tevatron and the LHC, respectively. (As above, although the muon chambers in the ATLAS detector at the LHC extend to $\sim 10 \mathrm{~m}$, those in the CMS detector only extend to $\sim 7 \mathrm{~m}$; we take the more conservative $7 \mathrm{~m}$ as our cut.) We again take $d_{\mathrm{pr}}=1 \mathrm{~cm}$. Each of these events produces a distinctive charged track with a kink due to the momentum carried away by the gravitino.

As above, both nonpointing and delayed events are included in this category. Furthermore, we include all generations $(e, \mu$, and $\tau)$. As mentioned previously, the stau is generally the lightest slepton, and hence we expect the majority of the decays in the 
stau-NLSP scenario to be of the form $\tilde{\tau} \rightarrow \tau \tilde{G}$. Although the heavier sleptons $\tilde{l}=(\tilde{e}$ or $\tilde{\mu})$ may also decay to $l \tilde{G}$, the branching ratio of this decay is generally suppressed compared to the decay to a lepton and a neutralino, i.e., $l \tilde{\chi}^{0}$. If the latter is kinematically forbidden, then the 3-body decays to $l \tau^{-} \tilde{\tau}^{+}$or $l \tau^{+} \tilde{\tau}^{-}$dominate instead. However, as the mass splitting between the stau NLSP and the heavier sleptons decreases, these 3-body decays become less dominant (becoming kinematically forbidden if the mass splitting becomes less than the tau mass). The decays to $l \tilde{G}$ may then occur if the heavier sleptons $\tilde{l}$ are produced at the end of a decay chain.

(4) Metastable sleptons (stau NLSP): Events in which at least one charged slepton passes through the entire detector before decaying to a charged lepton and a gravitino. That is, the gravitino is produced at $d_{\text {decay }} \geq d_{\mathrm{ms}}$, where $d_{\mathrm{ms}}$ is the distance to the outer edge of the detector. We take $d_{\mathrm{ms}}=5 \mathrm{~m}$ and $d_{\mathrm{ms}}=$ $10 \mathrm{~m}$ as conservative estimates of the sizes of the detectors at the Tevatron and the LHC, respectively. All generations $(\tilde{e}, \tilde{\mu}$, and $\tilde{\tau})$ are included. These events will produce charged tracks with a relatively large radius of curvature.

For this category, we impose a further cut, requiring that the speeds $\beta$ of the sleptons satisfy the criteria $\beta_{\text {lower }} \leq \beta \leq \beta_{\text {upper }}$. The lower cut removes slower sleptons, which may be identified with the incorrect collision event. The higher cut removes faster sleptons, which may be misidentified as muons. We take typical values $\beta_{\text {lower }}=0.6$ and $\beta_{\text {upper }}=0.8$. Note, however, that Ref. [69] suggests a new search strategy that may be sensitive to even higher values of $\beta$.

All of these events will also be distinguished by missing energy and momentum carried away by the gravitinos. Note that these categories are chosen to be illustrative of the variety of signals that may be observed, and that they are not comprehensive-we do not investigate prompt dilepton events or neutralino decays to $Z$ bosons, for example. Furthermore, the categories are not mutually exclusive; for example, one may easily have a single event in which both a nonprompt lepton and a metastable slepton are produced. It is also clear that the relevant detector systematics and backgrounds will also be different for each category. Finally, note that axino-LSP scenarios may have signals that are qualitatively similar to the very long-lived signatures discussed here; however, the gravitino-LSP and axino-LSP scenarios may be distinguished quantitatively by detailed studies of 3-body decay rates [79].

This categorization of events is somewhat oversimplified, as it is based primarily on cuts on the decay length. Certainly, additional cuts will be required in a realistic analysis, possibly reducing the number of detected signals. However, we shall soon see that these simple categories
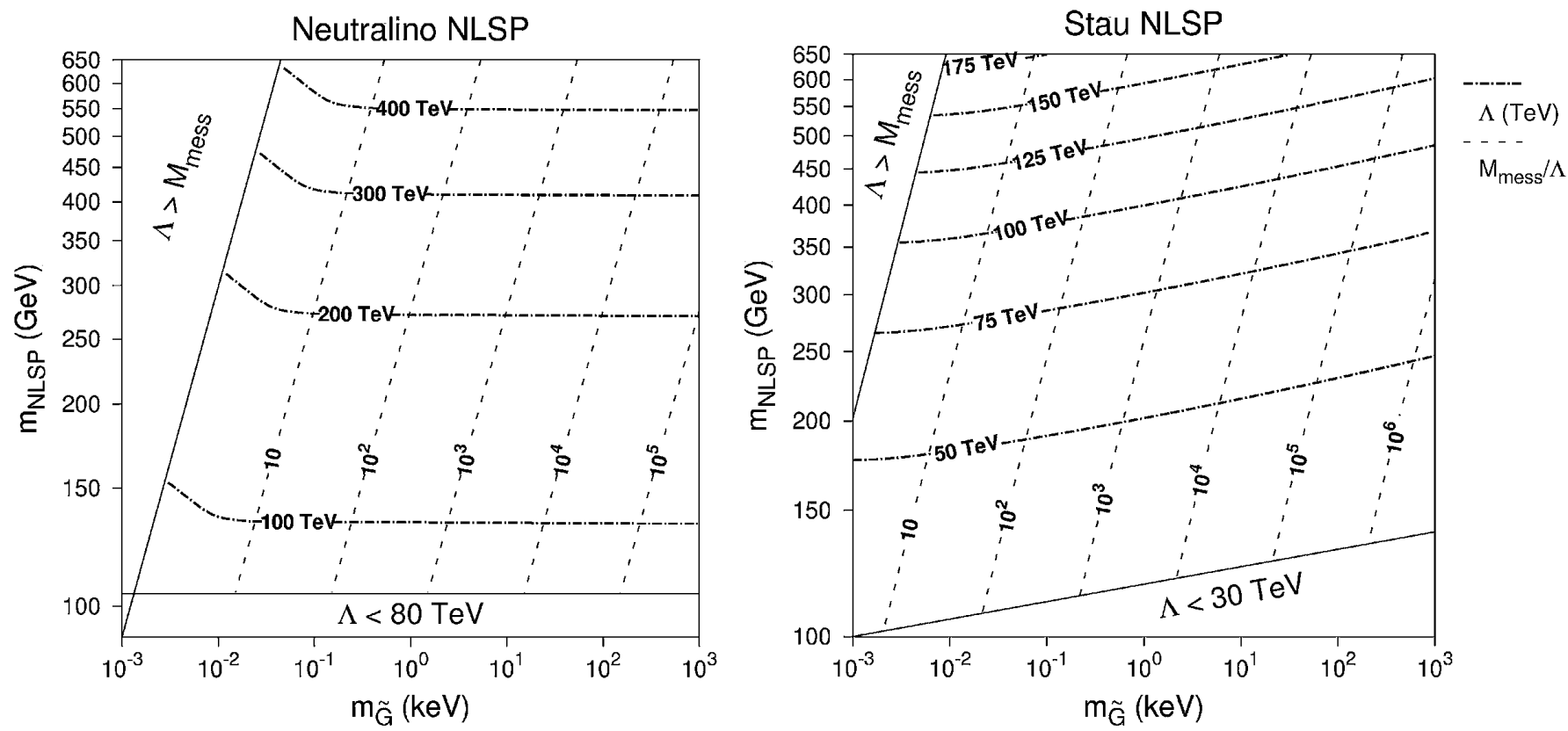

FIG. 1. Plots showing the mapping between the $m_{\tilde{G}}-m_{\mathrm{NLSP}}$ and the $M_{\text {mess }}-\Lambda$ GMSB parameter spaces, for the neutralino-NLSP scenario with $N_{5}=1$ (left) and stau-NLSP scenario with $N_{5}=4$ (right), where we fix $\tan \beta=20, \mu>0$, and $c_{\text {grav }}=1$ in both cases. Contours of constant $M_{\text {mess }} / \Lambda$ (dashed black line) and $\Lambda$ (dashed-dotted black line) are shown. The region in the upper-left corner is disallowed by theory, while the region at the bottom is excluded by experiment (using the conservative constraints mentioned in the text). 
align with the three cosmological scenarios outlined previously.

\section{B. GMSB scan and collider simulations}

We now calculate the event rates for these gravitino signals in a parametrized GMSB model. A large number of programs have been written for the numerical computation of the mass spectra and collider predictions for parametrized supersymmetric models $[80,81]$. In this paper, we use ISAJET 7.80/ISASUSY [82] to generate mass spectra and decay branching ratio tables. ISASUSY properly includes a number of 3-body decay processes relevant for gravitino phenomenology that are missing in other branching ratio programs.

ISAJET/ISASUSY takes values of the GMSB parameters listed in Eq. (15) as input. As discussed previously, here we focus on parametrizations that fix a subset of the GMSB parameters, resulting in either a neutralino or a stau NLSP. We then scan over $M_{\text {mess }}$ and $\Lambda$ (requiring that $M_{\text {mess }}>\Lambda$ ), resulting in spectra with a range of gravitino and NLSP masses. The correspondence between the $M_{\text {mess }}-\Lambda$ scan and the resulting $m_{\tilde{G}}-m_{\mathrm{NLSP}}$ parameter space is shown in Fig. 1.

We then take the spectra and decay tables output by ISAJET/ISASUSY and use them as input for the Monte Carlo
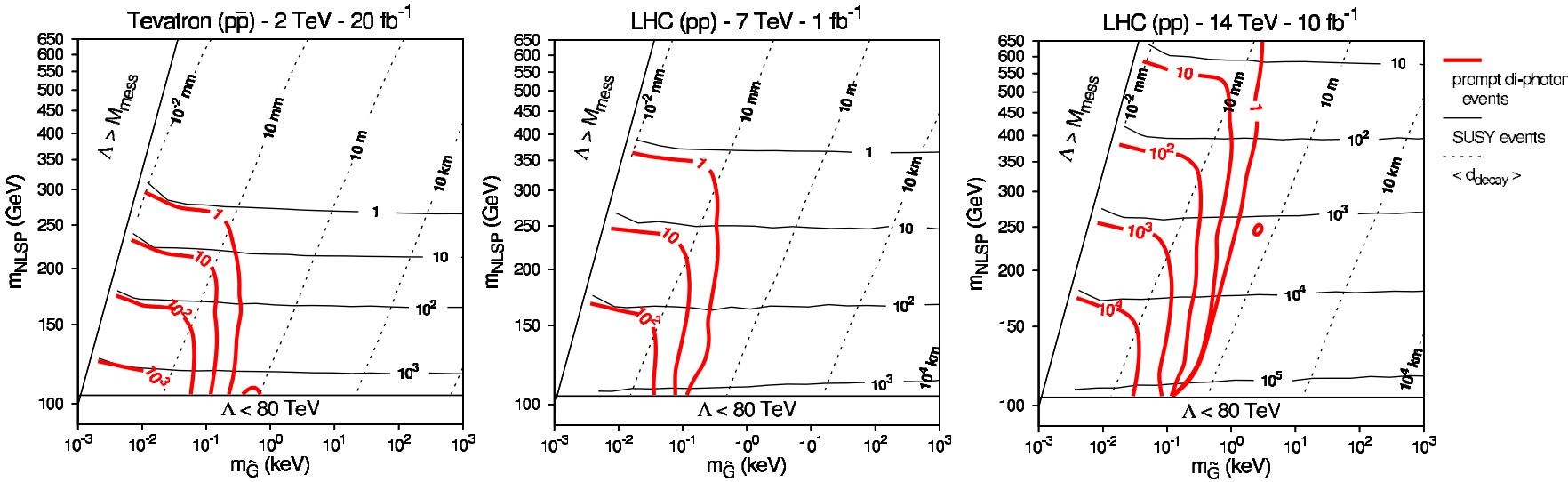

FIG. 2 (color online). Contour plots over the $m_{\tilde{G}}-m_{\mathrm{NLSP}}$ parameter space showing the expected number of prompt di-photon events (thick red line) in a model with a neutralino NLSP, for the three collider scenarios (indicated at the top of each plot) of interest. The total number of supersymmetric events (thin black line) and the average decay length $\left\langle d_{\text {decay }}\right\rangle$ (dashed black line) expected at each point in the parameter space are also indicated by contours. The region in the upper-left corner is disallowed if we require $\Lambda<M_{\text {mess }}$, while the region at the bottom is ruled out by LEP (using the conservative constraints discussed in the text). Note that hundreds of signal events may occur at the Tevatron with $20 \mathrm{fb}^{-1}$ and at the $7 \mathrm{TeV} \mathrm{LHC}$ with $1 \mathrm{fb}^{-1}$ if $m_{\tilde{G}} \lesssim$ tens of eV. Observation of such a number of events would suggest that the canonical thermal-production scenario is correct, and that light gravitinos compose only a fraction of the dark matter. Also, note that a larger fraction of neutralinos instead decay to $Z \tilde{G}$ as the neutralino mass increases (and that there is some small fraction of decays to $e^{+} e^{-} \tilde{G}$ and $\left.h \tilde{G}\right)$.
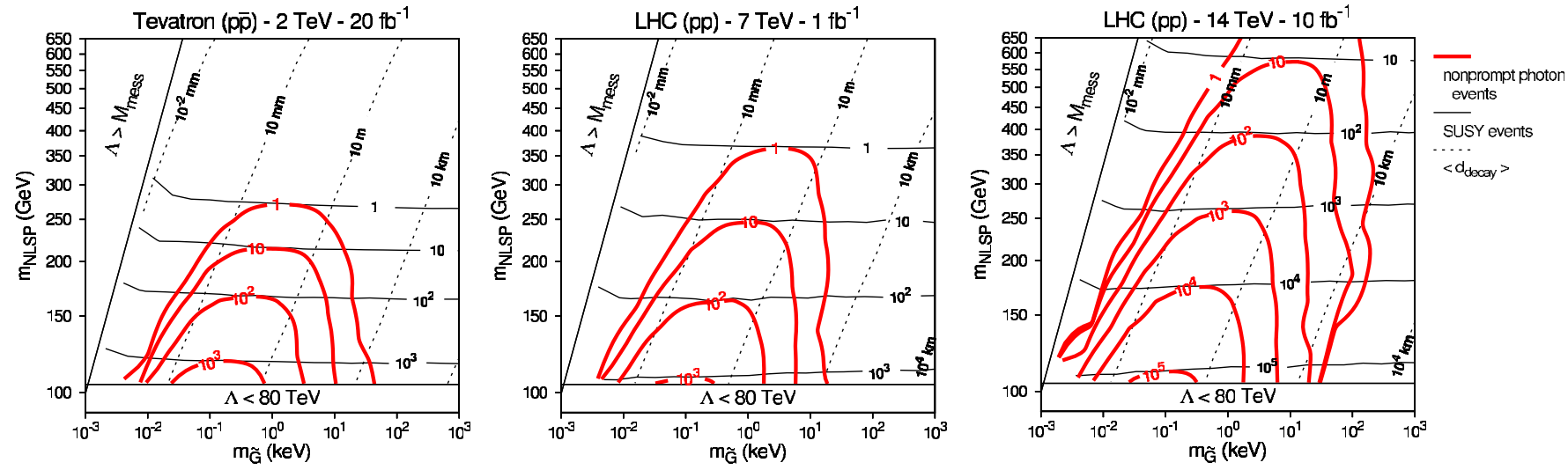

FIG. 3 (color online). The same as Fig. 2, but for nonprompt photon events. Note that hundreds of signal events may occur at the Tevatron with $20 \mathrm{fb}^{-1}$ and at the $7 \mathrm{TeV}$ LHC with $1 \mathrm{fb}^{-1}$ if tens of eV $\lesssim m_{\tilde{G}} \lesssim$ few keV. Observation of this number of events would suggest that a nonstandard cosmology and gravitino thermal history cooled relic gravitinos, and, for the top part of this mass range, also diluted the relic density. 
event generator PYTHIA 6.4.22 [83], including all supersymmetric processes available therein. For a given center-ofmass energy, PYTHIA can simulate a given number of collision events, giving a complete record of the various decay chains and final products generated in each event and an estimation of the various production cross sections. From this record, we can identify the supersymmetric "mother" particles that decay to directly produce gravitino and standard model "daughter" particles in each individual event. We can also find the decay length $d_{\text {decay }}$ away from the interaction point that each mother particle travels before decaying to produce a gravitino. Thus, for any number of simulated events, we can find the fraction that fall into each of the above categories. The expected number of signals from each category is then given by the respective fraction multiplied by the total number of supersym- metric events. We can also calculate the average $\left\langle d_{\text {decay }}\right\rangle$ of the decay length, taken over all supersymmetric events.

\section{Cosmological implications}

The results of the scan are shown in Figs. 2-5. We can see that the simple categorization of collider signals by decay-length cuts corresponds surprisingly well with the categorization of cosmological scenarios outlined previously. For example, Fig. 2 shows that the observation of hundreds of prompt events suggests that the first cosmological scenario $\left(m_{\tilde{G}} \lesssim 30 \mathrm{eV}\right)$ is likely to be valid. Likewise, the second cosmological scenario $(30 \mathrm{eV} \lesssim$ $m_{\tilde{G}} \lesssim$ few $\mathrm{keV}$ ) will be implied by the observation of a large number of nonprompt events, as demonstrated by Figs. 3 and 4. Finally, that the observation of a large number of metastable sleptons supports the third cosmo-
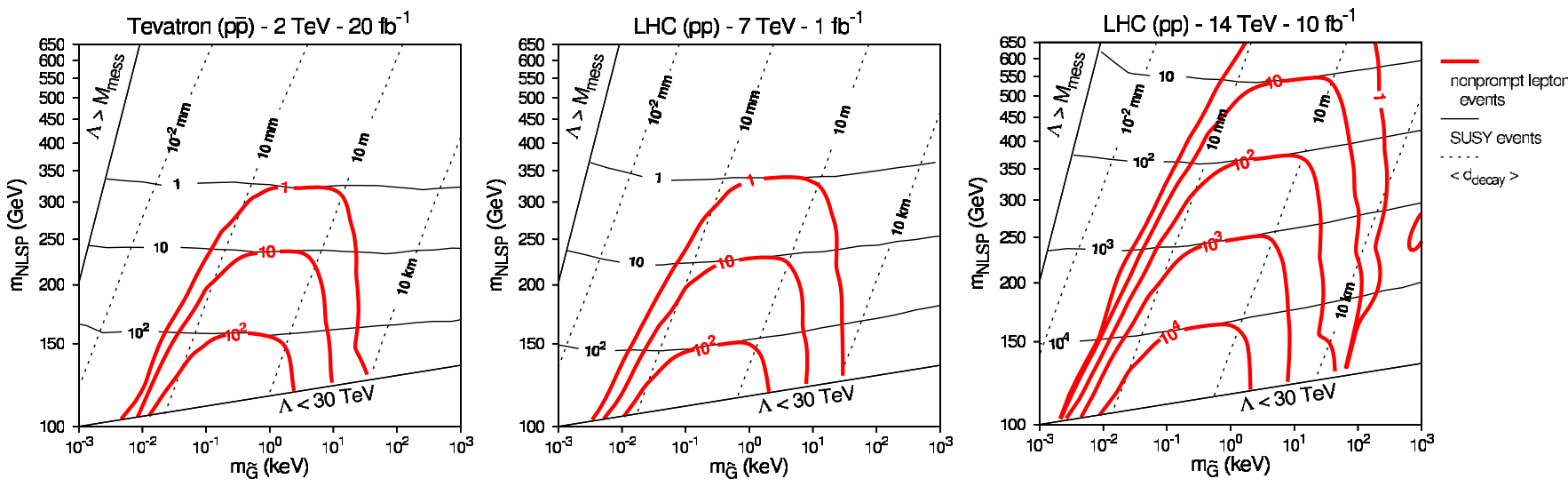

FIG. 4 (color online). Contour plots showing the expected number of nonprompt lepton events in a model with a stau NLSP. Note that hundreds of signal events may occur at the Tevatron with $20 \mathrm{fb}^{-1}$ and at the $7 \mathrm{TeV} \mathrm{LHC} \mathrm{with} 1 \mathrm{fb}^{-1}$ if tens of eV $\lesssim m_{\tilde{G}} \lessgtr$ few $\mathrm{keV}$. Observation of this number of events would suggest that a nonstandard cosmology and gravitino thermal history cooled relic gravitinos, and, for the top part of this mass range, also diluted the relic density.
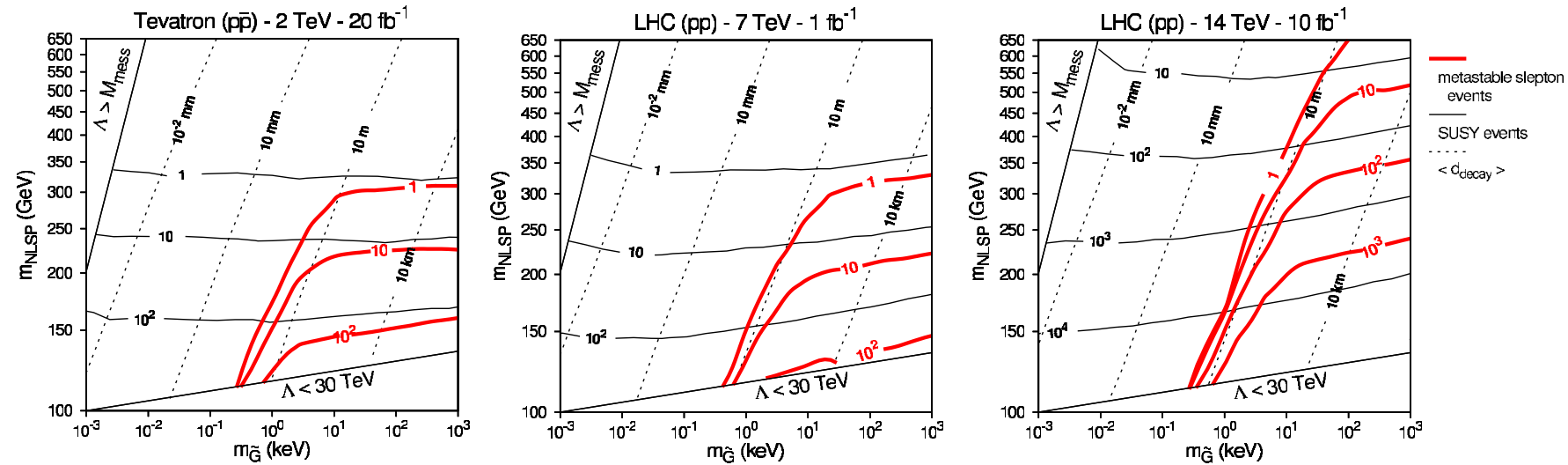

FIG. 5 (color online). The same as Fig. 4, but for metastable slepton events. Note that hundreds of signal events may occur at the Tevatron with $20 \mathrm{fb}^{-1}$ and at the $7 \mathrm{TeV}$ LHC with $1 \mathrm{fb}^{-1}$ if $m_{\tilde{G}} \gtrsim \mathrm{keV}$. Observation of such a number of events would suggest that gravitinos could entirely compose the dark matter, assuming some nonstandard cosmology diluted relic gravitinos. Also, note that although we only consider $m_{\tilde{G}} \lesssim \mathrm{MeV}$ here, GMSB models allow larger gravitino masses, up to $m_{\tilde{G}} \sim$ GeV. Thus, these plots may be straightforwardly extrapolated to higher gravitino masses if desired. However, note that at higher $M_{\text {mess }}$, and hence at higher $m_{\tilde{G}}$, the neutralino again becomes the NLSP; see Fig. 1 of Ref. [51]. 
logical scenario $\left(m_{\tilde{G}} \gtrsim\right.$ few $\left.\mathrm{keV}\right)$ can be seen in Fig. 5 . We emphasize that this correspondence is not strongly dependent on our specific choice of GMSB models. It is indeed a remarkable coincidence that theoretically motivated supersymmetric and gravitino-mass scales, the physical sizes of collider detectors, and gravitino cosmology all conspire to allow this correspondence.

Note also that we find that the number of gravitino events produced during the initial run of the LHC (center-of-mass energy of $7 \mathrm{TeV}$ and integrated luminosity of $1 \mathrm{fb}^{-1}$ ) may be comparable to that produced during an extended run of the Tevatron (center-of-mass energy of $2 \mathrm{TeV}$ and integrated luminosity of $20 \mathrm{fb}^{-1}$ ). This is true in regions of parameter space where large numbers of signals are expected. However, the higher center-of-mass energy of the LHC allows it to access regions of parameter space where $m_{\text {SUSY }}$ is larger; this is especially evident in the neutralino-NLSP scenario, as can be seen by comparing the left and middle panels in both Figs. 2 and 3.

If the distribution of mother-particle decay lengths can be measured with sufficient accuracy along with the total signal rate, then it may be possible to gain some information on the masses of the mother particles and the gravitino. To do so, it will be important to understand the distribution of energies and speeds with which mother particles are produced, since this will directly affect the distribution of decay lengths via dilation of the mother-particle lifetimes. In Fig. 6, we show some examples of probability distribution functions for the speed $\beta$ and the Lorentz factor $\gamma$ of mother particles that decay to gravitinos, for various collider scenarios.
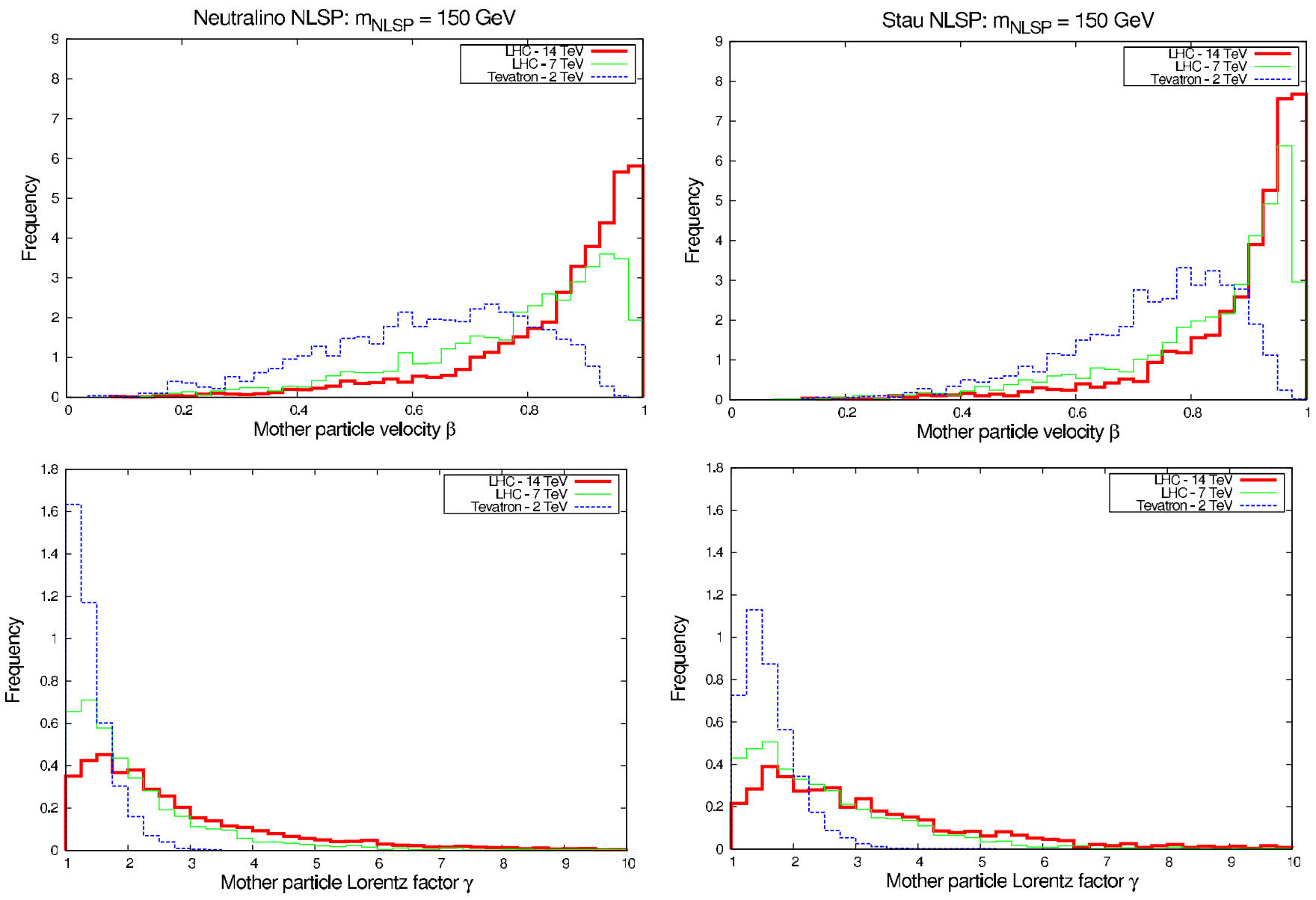

FIG. 6 (color online). Simulated probability distribution functions for the speed $\beta$ (top panels) and Lorentz factor $\gamma$ (bottom panels) of mother particles decaying to gravitinos, plotted for the three collider energies of interest, in the neutralino-NLSP (left) and stauNLSP (right) scenarios. We have chosen models with $m_{\mathrm{NLSP}}=150 \mathrm{GeV}$ for both NLSP scenarios (this was accomplished by choosing $\Lambda=115 \mathrm{TeV}$ in the neutralino-NLSP scenario and $\Lambda=40 \mathrm{TeV}$ in the stau-NLSP scenario, as well as setting $M_{\text {mess }} / \Lambda=10^{3}$ in both scenarios). The area under each curve has been normalized to unity. As expected, increasing the center-of-mass energy results in the production of faster mother particles. Note also that even though $m_{\mathrm{NLSP}}$ is identical for both of these scenarios, the staus are produced with slightly higher speeds. This is because the squark masses (which increase with increasing $\Lambda$ ) in the stau-NLSP scenario happen to be slightly lighter than those in the neutralino-NLSP scenario, for these choices of GMSB parameters. 


\section{CONCLUSIONS}

Light gravitinos in the mass range $\mathrm{eV}$ to $\mathrm{MeV}$ appear in GMSB models that naturally avoid flavor violation. We have examined the decay of supersymmetric particles to light gravitinos at colliders such as the Tevatron and the LHC. These decays will give rise to dramatic signatures, such as prompt di-photons or nonprompt photons, if the NLSP is a neutralino, or kinked charged tracks or heavy metastable charged particles, if the NLSP is a stau (or some other charged particle). We find large regions of the gravitino-mass-NLSP-mass parameter space in which the rate for such events may be appreciable at the Tevatron and LHC and which are consistent with current null supersymmetry searches.

Given that $m_{\tilde{G}} \ll m_{\mathrm{NLSP}}$ for these events, the decay kinematics of individual events cannot be used to determine the gravitino mass. However, the event rate and the distribution of decay locations may be used to narrow the range of NLSP and gravitino masses. Information about the nature of the NLSP may also be gleaned from the standard model decay products.

One of the attractions of supersymmetry has been its ability to provide a natural candidate for the cold dark matter required by a wealth of cosmological observations. Unfortunately, despite being well motivated in GMSB models, the canonical light-gravitino scenario does not provide a natural cold dark-matter candidate. Nevertheless, this canonical scenario does allow gravitinos with masses $m_{\tilde{G}} \lesssim 30 \mathrm{eV}$ that compose a fraction of the total dark matter, as determined by current astrophysical con- straints on the relic abundance and small-scale structure. Given that upcoming structure-formation observations are expected to probe hot dark-matter masses as low as $m_{\tilde{G}} \sim$ $\mathrm{eV}$, detection of a gravitino in the mass range $\mathrm{eV} \lesssim m_{\tilde{G}} \lesssim$ $30 \mathrm{eV}$ via prompt signals at colliders would have implications for future small-scale-structure measurements. And although masses $m_{\tilde{G}} \gtrsim 30 \mathrm{eV}$ are disfavored, they may still be possible if the pre-BBN history was different than in the canonical scenario. Detection of gravitinos in this mass range via nonprompt and metastable signals at colliders would thus have serious implications for earlyUniverse cosmology, and may provide some insight into the reheating and inflationary eras. And who knows? There may indeed be new early-Universe physics that results in a gravitino that has the right cosmological abundance and temperature to be the dark matter.

\section{ACKNOWLEDGMENTS}

S. K. L. thanks Maria Spiropulu and Sezen Sekmen for their assistance in setting up the collider simulations. S. K.L. and M.K. also thank Mark Wise for discussion of issues involving the gravitino production rate at low reheating temperatures. J.L.F. is grateful to William Molzon and Daniel Whiteson for helpful conversations. This work was supported at Caltech by the DOE DE-FG0392-ER40701 and the Gordon and Betty Moore Foundation. The work of J.L.F. was supported in part by NSF Grant No. PHY-0653656.
[1] D. V. Volkov and V. A. Soroka, Pis'ma Zh. Eksp. Teor. Fiz. 18, 529 (1973) [JETP Lett. 18, 312 (1973)].

[2] P. Fayet and J. Iliopoulos, Phys. Lett. 51B, 461 (1974).

[3] B. de Wit and D.Z. Freedman, Phys. Rev. Lett. 35, 827 (1975).

[4] S. Deser and B. Zumino, Phys. Rev. Lett. 38, 1433 (1977).

[5] M. Dine, W. Fischler, and M. Srednicki, Nucl. Phys. B189, 575 (1981).

[6] S. Dimopoulos and S. Raby, Nucl. Phys. B192, 353 (1981).

[7] C. R. Nappi and B. A. Ovrut, Phys. Lett. 113B, 175 (1982).

[8] L. Alvarez-Gaume, M. Claudson, and M. B. Wise, Nucl. Phys. B207, 96 (1982).

[9] M. Dine, A. E. Nelson, and Y. Shirman, Phys. Rev. D 51, 1362 (1995).

[10] M. Dine, A. E. Nelson, Y. Nir, and Y. Shirman, Phys. Rev. D 53, 2658 (1996).

[11] H. Pagels and J.R. Primack, Phys. Rev. Lett. 48, 223 (1982).

[12] M. Viel, J. Lesgourgues, M. G. Haehnelt, S. Matarrese, and A. Riotto, Phys. Rev. D 71, 063534 (2005).
[13] N. Cabibbo, G. R. Farrar, and L. Maiani, Phys. Lett. 105B, 155 (1981).

[14] S. Weinberg, Phys. Rev. Lett. 48, 1303 (1982).

[15] J. R. Ellis, J.E. Kim, and D. V. Nanopoulos, Phys. Lett. 145B, 181 (1984).

[16] T. Moroi, H. Murayama, and M. Yamaguchi, Phys. Lett. B 303, 289 (1993).

[17] M. Kawasaki and T. Moroi, Prog. Theor. Phys. 93, 879 (1995).

[18] S. Borgani, A. Masiero, and M. Yamaguchi, Phys. Lett. B 386, 189 (1996).

[19] M. Bolz, A. Brandenburg, and W. Buchmuller, Nucl. Phys. B606, 518 (2001); B790, 336(E) (2008).

[20] J. Pradler and F.D. Steffen, Phys. Rev. D 75, 023509 (2007).

[21] J. Pradler, arXiv:0708.2786.

[22] V.S. Rychkov and A. Strumia, Phys. Rev. D 75, 075011 (2007).

[23] E. Pierpaoli, S. Borgani, A. Masiero, and M. Yamaguchi, Phys. Rev. D 57, 2089 (1998).

[24] K. Ichikawa, M. Kawasaki, K. Nakayama, T. Sekiguchi, 
and T. Takahashi, J. Cosmol. Astropart. Phys. 08 (2009) 013.

[25] M. Viel, J. Lesgourgues, M. G. Haehnelt, S. Matarrese, and A. Riotto, Phys. Rev. Lett. 97, 071301 (2006).

[26] A. Boyarsky, J. Lesgourgues, O. Ruchayskiy, and M. Viel, J. Cosmol. Astropart. Phys. 05 (2009) 012.

[27] U. Seljak, A. Makarov, P. McDonald, and H. Trac, Phys. Rev. Lett. 97, 191303 (2006).

[28] J. R. Primack, New J. Phys. 11, 105029 (2009).

[29] S. Hannestad, Phys. Rev. D 70, 043506 (2004).

[30] T. Asaka, K. Hamaguchi, and K. Suzuki, Phys. Lett. B 490, 136 (2000).

[31] R. Allahverdi, A. Jokinen, and A. Mazumdar, Phys. Rev. D 71, 043505 (2005).

[32] E. J. Copeland and O. Seto, Phys. Rev. D 72, 023506 (2005).

[33] K. Kohri, T. Moroi, and A. Yotsuyanagi, Phys. Rev. D 73, 123511 (2006).

[34] F. D. Steffen, J. Cosmol. Astropart. Phys. 09 (2006) 001.

[35] J. Pradler and F. D. Steffen, Phys. Lett. B 648, 224 (2007).

[36] K. Y. Choi, L. Roszkowski, and R. Ruiz de Austri, J. High Energy Phys. 04 (2008) 016.

[37] K. Kohri, A. Mazumdar, and N. Sahu, Phys. Rev. D 80, 103504 (2009).

[38] D. Gorbunov, A. Khmelnitsky, and V. Rubakov, J. High Energy Phys. 12 (2008) 055.

[39] S. Dimopoulos, G. F. Giudice, and A. Pomarol, Phys. Lett. B 389, 37 (1996).

[40] K. Choi, K. Hwang, H. B. Kim, and T. Lee, Phys. Lett. B 467, 211 (1999).

[41] E. A. Baltz and H. Murayama, J. High Energy Phys. 05 (2003) 067.

[42] M. Fujii and T. Yanagida, Phys. Lett. B 549, 273 (2002).

[43] K. Jedamzik, M. Lemoine, and G. Moultaka, Phys. Rev. D 73, 043514 (2006).

[44] F. Staub, W. Porod, and J. Niemeyer, J. High Energy Phys. 01 (2010) 58.

[45] R. Kallosh, L. Kofman, A. D. Linde, and A. Van Proeyen, Phys. Rev. D 61, 103503 (2000).

[46] I. M. Shoemaker and A. Kusenko, Phys. Rev. D 80, 075021 (2009).

[47] A. de Gouvea, T. Moroi, and H. Murayama, Phys. Rev. D 56, 1281 (1997).

[48] R. Casalbuoni, S. De Curtis, D. Dominici, F. Feruglio, and R. Gatto, Phys. Lett. B 215, 313 (1988).

[49] T. Lee and G. H. Wu, Phys. Lett. B 447, 83 (1999).

[50] S. Ambrosanio, G. L. Kane, G. D. Kribs, S. P. Martin, and S. Mrenna, Phys. Rev. D 54, 5395 (1996).

[51] J. L. Feng and T. Moroi, Phys. Rev. D 58, 035001 (1998).

[52] H. Baer and X. Tata, Weak Scale Supersymmetry: From Superfields to Scattering Events (Cambridge University Press, Cambridge, 2006).

[53] G. D. Kribs, A. Martin, and T. S. Roy, J. High Energy Phys. 01 (2009) 023.
[54] Y. Santoso, AIP Conf. Proc. 1200, 494 (2010).

[55] D. R. Stump, M. Wiest, and C. P. Yuan, Phys. Rev. D 54, 1936 (1996).

[56] S. Dimopoulos, M. Dine, S. Raby, and S.D. Thomas, Phys. Rev. Lett. 76, 3494 (1996).

[57] S. Dimopoulos, S. D. Thomas, and J. D. Wells, Phys. Rev. D 54, 3283 (1996).

[58] P. Abreu et al. (DELPHI Collaboration), Eur. Phys. J. C 16, 211 (2000).

[59] H. Baer, P. G. Mercadante, X. Tata, and Y. L. Wang, Phys. Rev. D 62, 095007 (2000).

[60] S. Ambrosanio, B. Mele, S. Petrarca, G. Polesello, and A. Rimoldi, J. High Energy Phys. 01 (2001) 014.

[61] C. Pagliarone (CDF Collaboration and D0 Collaboration), arXiv:hep-ex/0312005.

[62] K. Hamaguchi, Y. Kuno, T. Nakaya, and M. M. Nojiri, Phys. Rev. D 70, 115007 (2004).

[63] J.L. Feng and B. T. Smith, Phys. Rev. D 71, 015004 (2005).

[64] P. Wagner and D. A. Toback, Int. J. Mod. Phys. A 20, 3267 (2005).

[65] H. U. Martyn, Eur. Phys. J. C 48, 15 (2006).

[66] M. Klasen and G. Pignol, Phys. Rev. D 75, 115003 (2007).

[67] K. Hamaguchi, S. Shirai, and T. T. Yanagida, Phys. Lett. B 663, 86 (2008).

[68] S. Tarem, S. Bressler, H. Nomoto, and A. Di Mattia, Eur. Phys. J. C 62, 281 (2009).

[69] J. Chen and T. Adams, Eur. Phys. J. C 67, 335 (2010).

[70] J. L. Feng, J. F. Grivaz, and J. Nachtman, Rev. Mod. Phys. 82, 699 (2010).

[71] A. Heister et al. (ALEPH Collaboration), Eur. Phys. J. C 25, 339 (2002).

[72] LEP SUSY Working Group (ALEPH, DELPHI, L3, and OPAL Collaborations), Report No. LEPSUSYWG/0209.2, http://lepsusy.web.cern.ch/lepsusy/Welcome.html.

[73] B. C. Allanach et al., Eur. Phys. J. C 25, 113 (2002).

[74] V. M. Abazov et al. (D0 Collaboration), Phys. Lett. B 659, 856 (2008).

[75] T. Aaltonen et al. (CDF Collaboration), Phys. Rev. Lett. 104, 011801 (2010).

[76] A. Abulencia et al. (CDF Collaboration), Phys. Rev. Lett. 99, 121801 (2007).

[77] V. M. Abazov et al. (D0 Collaboration), Phys. Rev. Lett. 102, 161802 (2009).

[78] T. Aaltonen et al. (CDF Collaboration), Phys. Rev. Lett. 103, 021802 (2009).

[79] A. Brandenburg, L. Covi, K. Hamaguchi, L. Roszkowski, and F. D. Steffen, Phys. Lett. B 617, 99 (2005).

[80] P. Skands et al., J. High Energy Phys. 07 (2004) 036.

[81] B. Allanach et al., Comput. Phys. Commun. 180, 8 (2009).

[82] F.E. Paige, S. D. Protopopescu, H. Baer, and X. Tata, arXiv:hep-ph/0312045. Documentation for ISAJET 7.80 can be found at http://www.nhn.ou.edu/ isajet/.

[83] T. Sjostrand, S. Mrenna, and P. Skands, J. High Energy Phys. 05 (2006) 026. 